\title{
First-order vortices in a gauged $C P(2)$ model with a Chern-Simons term
}

\author{
V. Almeida, ${ }^{1}$ R. Casana, ${ }^{1}$ and E. da Hora ${ }^{2}$ \\ ${ }^{1}$ Departamento de Física, Universidade Federal do Maranhão, 65080-805 São Luís, Maranhão, Brazil \\ ${ }^{2}$ Coordenadoria Interdisciplinar de Ciência e Tecnologia, Universidade Federal do Maranhão, \\ 65080-805 São Luís, Maranhão, Brazil
}

(Received 30 October 2017; published 25 January 2018)

\begin{abstract}
We consider a gauged $C P(2)$ theory in the presence of the Chern-Simons action, focusing our attention on those time-independent solutions possessing radial symmetry. In this context, we develop a coherent first-order framework via the Bogomol'nyi prescription, from which we obtain the corresponding energy lower bound and the first-order equations the model supports. We use these expressions to introduce effective Bogomol'nyi-Prasad-Sommerfeld (BPS) scenarios, solving the resulting first-order equations by means of the finite-difference scheme, this way attaining genuine field solutions engendering topological configurations. We depict the new profiles, commenting on the main properties they engender.
\end{abstract}

DOI: $10.1103 /$ PhysRevD.97.016013

\section{INTRODUCTION}

In the context of classical theories, solitons are described as those time-independent solutions arising within highly nonlinear models [1]. In this sense, vortices are radially symmetric solutions coming from planar scenarios in the presence of a gauge field.

Moreover, under very special circumstances, solitons can also be obtained via a set of first-order differential equations (instead of the second-order Euler-Lagrange ones), the resulting solutions minimizing the energy of the effective system [2].

In this sense, first-order vortices were first studied in the context of the simplest Maxwell-Higgs electrodynamics [3]. Furthermore, these solutions were verified to occur within the Chern-Simons-Higgs scenario, too [4]. Also, first-order vortices were recently considered in connection with nonstandard models [5], the resulting solutions being used as an attempt to explain some cosmological issues [6].

In such a context, it is especially interesting to consider the existence of well-behaved time-independent vortices arising from a $C P(N-1)$ scenario in the presence of a gauge field, mainly due to the close phenomenological relation between such theory and the four-dimensional Yang-Mills-Higgs one [7].

In a recent investigation, radially symmetric solutions arising from a planar $C P(2)$ theory endowed by the Maxwell term were considered, the author clarifying the

Published by the American Physical Society under the terms of the Creative Commons Attribution 4.0 International license. Further distribution of this work must maintain attribution to the author(s) and the published article's title, journal citation, and DOI. Funded by SCOAP . way these structures and correlated results depend on the parameters of the model [8]. In that work, however, the vortex configurations were obtained by solving the secondorder Euler-Lagrange equations directly (the resulting solutions therefore not saturating the Bogomol'nyi bound).

In the sequel, some of us introduced the first-order vortices inherent to the aforementioned Maxwell $C P(2)$ theory, defining the energy lower bound and the corresponding first-order equations [9]. In that work, the selfdual profiles were constructed numerically by means of the finite-difference scheme, the resulting structures presenting the typical topological shape.

Moreover, some of us have also studied first-order vortices within a Maxwell $C P(2)$ model in the presence of a nontrivial dielectric function. The point to be raised here is that such a function can be used to change the vacuum manifold of the effective theory, from which we have used such freedom to generate self-dual vortices engendering a nontopological profile, the resulting Bogomol'nyi bound being not quantized anymore [10].

We now go a little bit further by investigating a rather natural extension of the aforecited works, i.e., the search for the first-order planar solitons arising from a $C P(2)$ theory in the presence of the Chern-Simons action.

To introduce our results, the present article is organized as follows: in Sec. II, we define the gauged $C P(N-1)$ theory and some conveniences inherent to it, focusing our attention on those time-independent solitons possessing radial symmetry. We then develop a coherent first-order framework by minimizing the effective energy according to the Bogomol'nyi prescription, this way obtaining general first-order equations and the corresponding energy lower bound, such construction only being possible due to a differential constraint involving the potential engendering 
self-duality. In Sec. III, we solve the first-order expressions in order to find genuine Bogomol'nyi-Prasad-Sommerfeld (BPS) solutions saturating the Bogomol'nyi bound. We solve the corresponding first-order equations by means of the finite-difference algorithm, from which we depict the numerical solutions, while commenting the main properties they engender. We end our work in Sec. IV, presenting our final considerations and the perspectives regarding future studies.

In this article, we adopt $\eta^{\mu \nu}=(+--)$ as the metric signature for the flat spacetime, together with the natural units system, for the sake of simplicity.

\section{THE MODEL}

We begin our investigation by presenting the Lagrange density defining the gauged $C P(N-1)$ model in the presence of the Chern-Simons term (with $\epsilon^{012}=+1$ ), i.e.,

$L=-\frac{k}{4} \epsilon^{\alpha \mu \nu} A_{\alpha} F_{\mu \nu}+\left(P_{a b} D_{\mu} \phi_{b}\right)^{*} P_{a c} D^{\mu} \phi_{c}-V(|\phi|)$.

Here, $F_{\mu \nu}=\partial_{\mu} A_{\nu}-\partial_{\nu} A_{\mu}$ stands for the electromagnetic field strength tensor, $D_{\mu} \phi_{a}=\partial_{\mu} \phi_{a}-i g A_{\mu} Q_{a b} \phi_{b}$ representing the covariant derivative $\left(Q=\left[Q_{a b}\right]\right.$ is a real diagonal charge matrix with null trace, i.e., $\operatorname{Tr} Q=0$ ). Furthermore, $P_{a b}=\delta_{a b}-h^{-1} \phi_{a} \phi_{b}^{*}$ is a projection operator defined conveniently. In this work, the Greek indices run over the space-time coordinates, the Latin ones counting the complex fields underlying the $C P(N-1)$ sector (with $\phi_{a}^{*} \phi_{a}=h$ ).

The model (1) is invariant under the global $S U(N)$ transformations

$$
\phi_{a}(x) \rightarrow U_{a b} \phi_{b}(x)
$$

and the local $U(1)$ ones

$$
\phi_{a}(x) \rightarrow \exp [i \Lambda(x)] \phi_{a}(x) .
$$

In addition, (1) possesses also a local $U(1)$ gauge symmetry, i.e.,

$$
\begin{gathered}
A_{\mu}(x) \rightarrow A_{\mu}(x)+\frac{1}{g} \partial_{\mu} \tilde{\Lambda}, \\
\phi_{a}(x) \rightarrow \exp \left[i q_{a} \tilde{\Lambda}(x)\right] \phi_{a}(x),
\end{gathered}
$$

where $q_{a}$ is the diagonal element $Q_{a a}$ of the charge matrix $Q$.

The Euler-Lagrange equation for the gauge field is given by

$$
\frac{k}{2} \epsilon^{\lambda \mu \nu} F_{\mu \nu}=J^{\lambda},
$$

where (H.c. means the Hermitian conjugate)

$$
J^{\lambda}=i g\left[\left(P_{a b} Q_{b c} \phi_{c}\right)^{*} P_{a d} D^{\lambda} \phi_{d}-\text { H.c. }\right]
$$

represents the current vector.

It is then instructive to write down the Gauss law for time-independent configurations, which reads (here, $B=F_{21}$ is the magnetic field)

$$
k B=\rho,
$$

where

$$
\rho=-2 g^{2} A^{0} \phi_{m}^{*} Q_{m b} P_{b c} Q_{c d} \phi_{d}
$$

is the electric charge density. In view of (8) and (9), the gauge $A^{0}=0$ cannot be chosen since it leads to trivial solutions everywhere. In other words, the first-order profiles we consider in this article make sense only when $A^{0} \neq 0$, the final structures possessing both nontrivial electric and magnetic fields.

In this work, we look for radially symmetric solutions inherent to the gauged $C P(2)$ scenario by using the usual map

$$
\begin{gathered}
A_{i}=-\frac{1}{g r} \epsilon^{i j} n^{j} A(r), \\
\left(\begin{array}{c}
\phi_{1} \\
\phi_{2} \\
\phi_{3}
\end{array}\right)=h^{\frac{1}{2}}\left(\begin{array}{c}
e^{i m_{1} \theta} \sin (\alpha(r)) \cos (\beta(r)) \\
e^{i m_{2} \theta} \sin (\alpha(r)) \sin (\beta(r)) \\
e^{i m_{3} \theta} \cos (\alpha(r))
\end{array}\right),
\end{gathered}
$$

with $m_{1}, m_{2}$, and $m_{3} \in \mathbb{Z}$ standing for winding numbers, $\epsilon^{i j}$ being the bidimensional Levi-Cività tensor (with $\left.\epsilon^{12}=+1\right)$, and $n^{j}=(\cos \theta, \sin \theta)$ representing the unit vector position. Therefore, regular solutions presenting no divergences are obtained via those profile functions $\alpha(r)$ and $A(r)$ satisfying

$$
\alpha(r \rightarrow 0) \rightarrow 0 \quad \text { and } \quad A(r \rightarrow 0) \rightarrow 0 .
$$

Now, concerning the combination between the charge matrix $Q_{a b}$ and the winding numbers appearing in (11), there are two possible choices supporting topological solitons [8]: (i) $Q=\lambda_{3} / 2$ and $m_{1}=-m_{2}=m$, and (ii) $Q=\lambda_{8} / 2$ and $m_{1}=m_{2}=m$ [both ones with $m_{3}=0, \lambda_{3}$ and $\lambda_{8}$ standing for the diagonal Gell-Mann matrices: $\lambda_{3}=\operatorname{diag}(1,-1,0)$ and $\left.\sqrt{3} \lambda_{8}=\operatorname{diag}(1,1,-2)\right]$. However, in Ref. [8], the author has demonstrated that these two combinations simply mimic each other, therefore only one effective scenario existing. In this sense, we consider only the case defined by $m_{1}=-m_{2}=m, m_{3}=0$, and

$$
Q_{a b}=\frac{1}{2} \lambda_{3}=\frac{1}{2} \operatorname{diag}(1,-1,0),
$$

the Euler-Lagrange equation for the profile function $\beta(r)$ reading 


$$
\frac{d^{2} \beta}{d r^{2}}+\left(\frac{1}{r}+2 \cot \alpha \frac{d \alpha}{d r}\right) \frac{d \beta}{d r}=H \sin ^{2} \alpha \sin (4 \beta),
$$

where

$$
H(r)=\frac{1}{r^{2}}\left(m-\frac{A}{2}\right)^{2}-\frac{g^{2}\left(A_{0}\right)^{2}}{4} \sin ^{2} \alpha
$$

is an auxiliary function, the solutions for $\beta(r)$ being

$$
\beta(r)=\beta_{1}=\frac{\pi}{4}+\frac{\pi}{2} k \quad \text { or } \quad \beta(r)=\beta_{2}=\frac{\pi}{2} k,
$$

with $k \in \mathbb{Z}$.

It is important to highlight that, from this point on, our expressions describe the effective scenario defined by the conveniences introduced in the previous paragraph.

In addition, we clarify that, in view of the radially symmetric map (10) and (11), the Gauss law (8) can be explicitly written as

$$
A^{0}=-\frac{2 k B}{g^{2} h W}
$$

where

$$
\rho=-\frac{g^{2} h}{2} A^{0} W
$$

stands for the charge density. Here, we have defined

$$
W=W(\alpha, \beta)=\sin ^{2} \alpha\left(1-\sin ^{2} \alpha \cos ^{2}(2 \beta)\right),
$$

with (17) being the equation of motion for $A^{0}(r)$ [i.e., the zeroth component of the Euler-Lagrange equation (6)]. Therefore, given the solutions to the profile function $\alpha(r)$ and the magnetic field $B(r)$, the electric potential $A^{0}(r)$ can be calculated directly from (17).

We look for genuine first-order solutions saturating an energy lower bound. In this sense, we proceed with the minimization of the overall energy, the starting point being the energy-momentum tensor related to the effective scenario, i.e.,

$$
T_{\lambda \rho}=\left(\left(P_{a b} D_{\lambda} \phi_{b}\right)^{*} P_{a c} D_{\rho} \phi_{c}+\text { H.c. }\right)-\eta_{\lambda \rho} L_{\text {ntop }},
$$

where

$$
L_{\text {ntop }}=\left(P_{a b} D_{\mu} \phi_{b}\right)^{*} P_{a c} D^{\mu} \phi_{c}-V(|\phi|)
$$

stands for the nontopological Lagrange density, the energy density $\varepsilon=T_{00}$ reading

$$
\varepsilon=\left(P_{a b} D_{0} \phi_{b}\right)^{*} P_{a c} D_{0} \phi_{c}+\left(P_{a b} D_{j} \phi_{b}\right)^{*} P_{a c} D_{j} \phi_{c}+V .
$$

Moreover, we use (10) and (11) in order to calculate

$$
\left(P_{a b} D_{0} \phi_{b}\right)^{*} P_{a c} D_{0} \phi_{c}=\frac{g^{2} h}{4}\left(A^{0}\right)^{2} W
$$

$$
\left(P_{a b} D_{j} \phi_{b}\right)^{*} P_{a c} D_{j} \phi_{c}=h\left[\left(\frac{d \alpha}{d r}\right)^{2}+\frac{W}{r^{2}}\left(\frac{A}{2}-m\right)^{2}\right],
$$

via which we rewrite (22) as

$$
\varepsilon=\frac{g^{2} h}{4}\left(A^{0}\right)^{2} W+h\left[\left(\frac{d \alpha}{d r}\right)^{2}+\frac{W}{r^{2}}\left(\frac{A}{2}-m\right)^{2}\right]+V .
$$

We now follow the standard procedure regarding the Chern-Simons models; i.e., we use the Gauss law (17) in order to write down the first term in (25) in terms of the magnetic field $B(r)$. In this sense, the energy distribution (25) reduces to

$$
\varepsilon=\frac{k^{2} B^{2}}{g^{2} h W}+h\left[\left(\frac{d \alpha}{d r}\right)^{2}+\frac{W}{r^{2}}\left(\frac{A}{2}-m\right)^{2}\right]+V,
$$

via which we proceed with the minimization of the total energy according to the Bogomol'nyi approach [2].

The point to be raised is that, whether the potential is constrained to satisfy

$$
\frac{2 k}{g^{2} \sqrt{h}} \frac{d}{d r} \sqrt{\frac{V}{W}}=-h \sqrt{W} \frac{d \alpha}{d r},
$$

the expression for the energy density can be rewritten according to the Bogomol'nyi prescription, therefore giving rise to

$$
\begin{aligned}
\varepsilon= & \left(\frac{k B}{g \sqrt{h W}} \mp \sqrt{V}\right)^{2}+h\left(\frac{d \alpha}{d r} \mp \frac{\sqrt{W}}{r}\left(\frac{A}{2}-m\right)\right)^{2} \\
& \mp \frac{2 k}{g^{2} \sqrt{h}} \frac{1}{r} \frac{d}{d r}\left[(A-2 m) \sqrt{\frac{V}{W}}\right],
\end{aligned}
$$

where we have used

$$
B(r)=-\frac{A^{\prime}}{g r}
$$

for the magnetic field (the prime denoting the derivative with respect to $r$ ), the resulting first-order equations standing for

$$
\begin{gathered}
\frac{d \alpha}{d r}= \pm \frac{\sqrt{W}}{r}\left(\frac{A}{2}-m\right), \\
k B= \pm g \sqrt{h V W}
\end{gathered}
$$


the solution for $\beta(r)$ being necessarily one of those stated in (16).

The scenario can be summarized as follows: given the potential fulfilling the constraint (27), the model (1) effectively supports radially symmetric solutions satisfying the first-order equations (30) and (31), the final configurations saturating an energy lower bound given by

$$
E_{b p s}=2 \pi \int r \varepsilon_{b p s} d r=\mp \frac{8 \pi m k}{g^{2} \sqrt{h}} \sqrt{\frac{V_{0}}{W_{0}}},
$$

where

$$
\varepsilon_{b p s}=\mp \frac{2 k}{g^{2} \sqrt{h}} \frac{1}{r} \frac{d}{d r}\left[(A-2 m) \sqrt{\frac{V}{W}}\right]
$$

stands for the energy density of the first-order structures, the upper (lower) sign holding for negative (positive) values of $m$. Here, we have supposed that $\left(A_{\infty}-2 m\right) \sqrt{V_{\infty} / W_{\infty}}$ vanishes, with $\sqrt{V_{0} / W_{0}}$ being finite. Moreover, we have defined $V_{0} \equiv V(r \rightarrow 0), \quad W_{0} \equiv W(r \rightarrow 0), \quad V_{\infty} \equiv$ $V(r \rightarrow \infty), W_{\infty} \equiv W(r \rightarrow \infty)$, and $A_{\infty} \equiv A(r \rightarrow \infty)$.

It is also instructive to calculate the magnetic flux $\Phi_{B}$ the first-order solutions support. It reads

$$
\Phi_{B}=2 \pi \int r B(r) d r=-\frac{2 \pi}{g} A_{\infty},
$$

where we have used $B(r)=-A^{\prime} / g r$ again. We demonstrate below that the energy lower bound (32) can be verified to be proportional to the magnetic flux (34), both quantities being quantized according to the winding number $m$, as expected for topological solitons.

\section{FIRST-ORDER SCENARIOS AND THEIR NUMERICAL SOLUTIONS}

We now demonstrate how the first-order framework we have developed generates genuine radially symmetric solitons. Here, to present our results, we proceed as follows: first, we choose a particular solution for $\beta(r)$ coming from (16), while solving the constraint (27) for the potential engendering self-duality. We then use such conveniences to obtain the asymptotic boundary conditions $\alpha(r)$ and $A(r)$ must obey in order to fulfill the finite-energy requirement, i.e., $\varepsilon(r \rightarrow \infty) \rightarrow 0$, from which we also calculate the energy lower bound (32) and the magnetic flux (34) explicitly, showing that they are proportional to each other, as expected. Finally, we solve the corresponding first-order equations numerically by means of the finite-difference scheme, while commenting on the main properties they engender.

\section{A. The $\beta(r)=\beta_{1}$ case}

We go further into our investigation by choosing

$$
\beta(r)=\beta_{1}=\frac{\pi}{4}+\frac{\pi}{2} k,
$$

from which one gets $\cos ^{2}\left(2 \beta_{1}\right)=0$, the fundamental constraint being reduced to

$$
\frac{2 k}{g^{2} \sqrt{h}} \frac{d}{d r}\left[\frac{\sqrt{V}}{\sin \alpha}\right]=h \frac{d}{d r}(\cos \alpha),
$$

whose solution is

$$
V(\alpha)=\frac{g^{4}}{16 k^{2}} h^{3} \sin ^{2}(2 \alpha)
$$

or

$$
V\left(\left|\phi_{3}\right|\right)=\frac{g^{4} h}{4 k^{2}}\left|\phi_{3}\right|^{2}\left(h-\left|\phi_{3}\right|^{2}\right),
$$

i.e., the potential supporting self-duality (here, we have used $C=0$ for the integration constant).

We now implement (35) and (37) into (26), the resulting expression being

$$
\begin{aligned}
\varepsilon(r)= & \frac{k^{2} B^{2}}{g^{2} h \sin ^{2} \alpha}+\frac{g^{4}}{16 k^{2}} h^{3} \sin ^{2}(2 \alpha) \\
& +h\left[\left(\frac{d \alpha}{d r}\right)^{2}+\frac{\sin ^{2} \alpha}{r^{2}}\left(\frac{A}{2}-m\right)^{2}\right],
\end{aligned}
$$

from which we attain $\varepsilon(r \rightarrow \infty) \rightarrow 0$ by imposing

$$
\alpha(r \rightarrow \infty) \rightarrow \frac{\pi}{2} \quad \text { and } \quad A(r \rightarrow \infty) \rightarrow 2 m,
$$

standing for the boundary conditions the profile functions obey in the asymptotic limit. Here, it is particularly interesting to note that, given the condition $\alpha(r \rightarrow \infty)$ $\rightarrow \pi / 2$, the potential (38) spontaneously breaks the original $S U(3)$ symmetry into the $S U(2)$ one.

In view of (35), (37), and (40), the energy lowerbound (32) can be verified to be equal to

$$
E_{b p s}=\mp 4 \pi h m,
$$

the magnetic flux $\Phi_{B}$ (34) standing for

$$
\Phi_{B}=-\frac{4 \pi}{g} m,
$$

from which we get that $E_{b p s}= \pm g h \Phi_{B}$, both $E_{b p s}$ and $\Phi_{B}$ being proportional to each other and quantized according to the winding number $m$, as expected. Here, we have used

$$
\frac{\sqrt{V_{0}}}{\sin \alpha_{0} \sqrt{1-\sin ^{2} \alpha_{0} \cos ^{2}\left(2 \beta_{1}\right)}}=\frac{g^{2} \sqrt{h}}{2 k} h,
$$

this way also verifying our previous assumption; see the discussion just after (33). 
We also calculate, for completeness, the total electric charge $Q_{e}$ the first-order vortices carry by integrating the Gauss law (8). It gives

$$
Q_{e}=2 \pi \int r \rho(r) d r=k \Phi_{B}
$$

where $\Phi_{B}$ is given by (42).

The first-order equations (30) and (31) can be rewritten as

$$
\begin{gathered}
\frac{d \alpha}{d r}= \pm \frac{\sin \alpha}{r}\left(\frac{A}{2}-m\right), \\
\frac{1}{r} \frac{d A}{d r}=\mp \frac{g^{4}}{4 k^{2}} h^{2} \sin (2 \alpha) \sin \alpha,
\end{gathered}
$$

which must be solved according to the boundary conditions (12) and (40).

\section{B. The $\beta(r)=\beta_{2}$ case}

We now consider

$$
\beta(r)=\beta_{2}=\frac{\pi}{2} k,
$$

via which one gets $\cos ^{2}\left(2 \beta_{2}\right)=1$, the corresponding constraint being

$$
\frac{4 k}{g^{2} \sqrt{h}} \frac{d}{d r}\left[\frac{\sqrt{V}}{\sin (2 \alpha)}\right]=\frac{h}{4} \frac{d}{d r}(\cos (2 \alpha)),
$$

the solution standing for the self-dual potential, i.e.,

$$
V(\alpha)=\frac{g^{4}}{16 k^{2}}\left(\frac{h}{4}\right)^{3} \sin ^{2}(4 \alpha)
$$

or

$$
V\left(\left|\phi_{3}\right|\right)=\frac{g^{4}}{64 k^{2} h}\left|\phi_{3}\right|^{2}\left(h-\left|\phi_{3}\right|^{2}\right)\left(h-2\left|\phi_{3}\right|^{2}\right)^{2},
$$

where we have chosen $C_{1}=0$ for the integration constant.

We proceed in the very same way as before; i.e., we use (47) and (49) into (26), from which one gets the general expression

$$
\begin{aligned}
\varepsilon(r)= & \frac{4 k^{2} B^{2}}{g^{2} h \sin ^{2}(2 \alpha)}+\frac{g^{4}}{16 k^{2}}\left(\frac{h}{4}\right)^{3} \sin ^{2}(4 \alpha) \\
& +h\left[\left(\frac{d \alpha}{d r}\right)^{2}+\frac{\sin ^{2}(2 \alpha)}{4 r^{2}}\left(\frac{A}{2}-m\right)^{2}\right],
\end{aligned}
$$

the finite-energy requirement $\varepsilon(r \rightarrow \infty) \rightarrow 0$ being attained by those profile functions fulfilling

$$
\alpha(r \rightarrow \infty) \rightarrow \frac{\pi}{4} \quad \text { and } \quad A(r \rightarrow \infty) \rightarrow 2 m,
$$

i.e., the boundary conditions in the limit $r \rightarrow \infty$.

Now, because of (47), (49), and (52), the energy bound (32) reduces to

$$
E_{b p s}=\mp \pi h m,
$$

the magnetic flux $\Phi_{B}$ and the total electric charge $Q_{e}$ still being given by (42) and (44), respectively. Therefore, one gets that $E_{b p s}= \pm g h \Phi_{B} / 4$, the lower bound being proportional to the flux of the magnetic field, both ones being again quantized. Here, we have calculated

$$
\frac{\sqrt{V_{0}}}{\sin \alpha_{0} \sqrt{1-\sin ^{2} \alpha_{0} \cos ^{2}\left(2 \beta_{2}\right)}}=\frac{g^{2} \sqrt{h}}{2 k} \frac{h}{4}
$$

in order to verify our previous conjecture.

In this case, the first-order expressions (30) and (31) can be written in the form

$$
\begin{gathered}
\frac{d \alpha}{d r}= \pm \frac{\sin (2 \alpha)}{2 r}\left(\frac{A}{2}-m\right), \\
\frac{1}{r} \frac{d A}{d r}=\mp \frac{g^{4}}{4 k^{2}}\left(\frac{h}{4}\right)^{2} \sin (4 \alpha) \sin (2 \alpha),
\end{gathered}
$$

which must be considered in the presence of the conditions (12) and (52).

It is worthwhile to point out that Eqs. (55) and (56) can be obtained directly from those in (45) and (46) via the redefinitions $\alpha \rightarrow 2 \alpha$ and $h \rightarrow h / 4$, the energy bound and the self-dual potential behaving in a similar way, the magnetic flux remaining the same. Therefore, given that the two first-order scenarios introduced above are phenomenologically equivalent, one concludes with the existence of only one effective scenario. In this sense, from now on, we focus our attention on those expressions coming from $\beta(r)=\beta_{1}$ only.

In what follows, we depict the results we have found by solving the first-order equations (45) and (46) by means of the finite-difference prescription, according to the boundary conditions (12) and (40). Here, we have considered the lower signs in the first-order expressions (i.e., $m>0$ only), while choosing $h=k=1$ and $g=\sqrt{2}$, for the sake of simplicity. In this sense, we depict the solutions to the profile functions $\alpha(r)$ and $A(r)$ themselves, the magnetic field $B(r)$ (29), the BPS energy density $\varepsilon_{b p s}(r)$ (33), the electric potential $A^{0}(r)(17)$, and the electric field $E(r)=$ $-d A^{0} / d r$ for $m=1$ (solid black line), $m=2$ (dashed blue line), and $m=3$ (dash-dotted red line).

In Figs. 1 and 2, we plot the numerical profiles to the functions $\alpha(r)$ and $A(r)$, respectively, from which we verify the monotonic manner these fields use to approach 


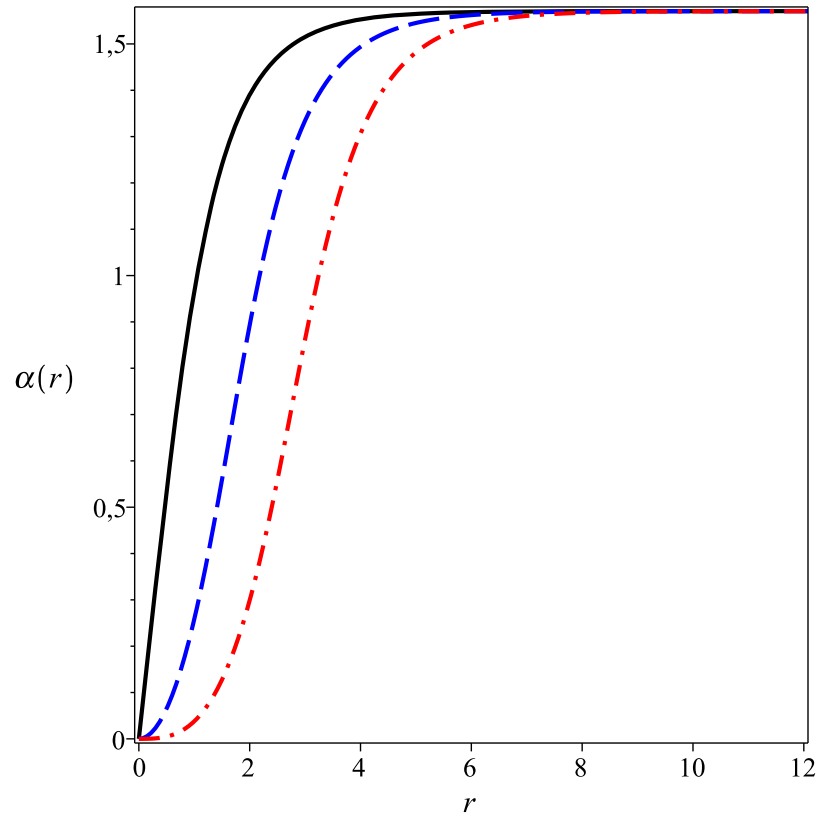

FIG. 1. Numerical solutions to $\alpha(r)$ coming from (45) and (46) in the presence of (12) and (40). Here, we have fixed $h=k=1$ and $g=\sqrt{2}$, varying the winding number: $m=1$ (solid black line), $m=2$ (dashed blue line), and $m=3$ (dash-dotted red line).

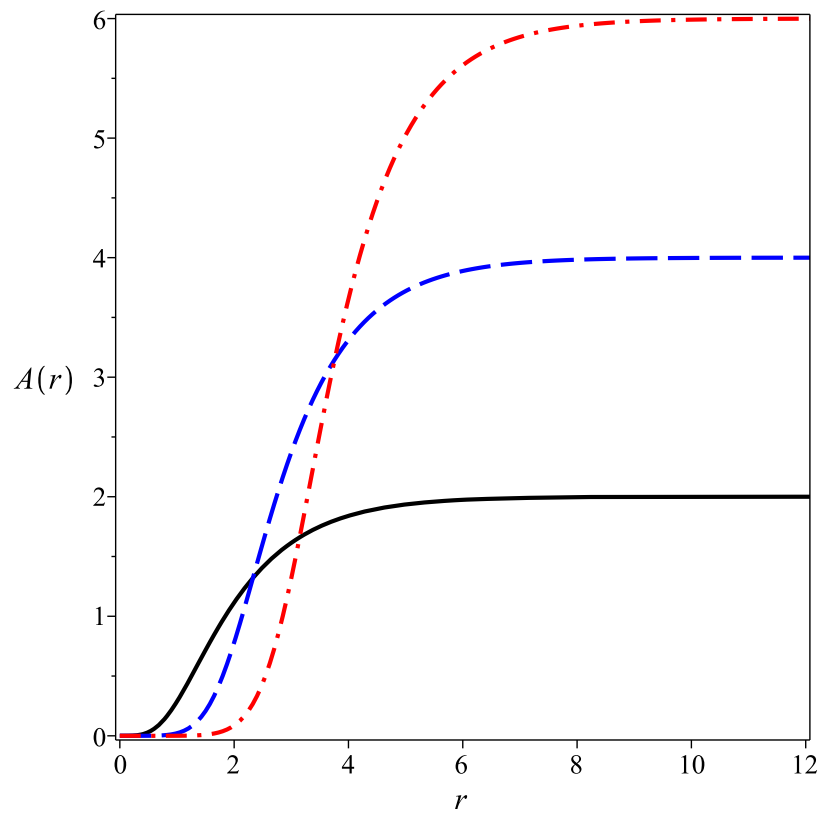

FIG. 2. Numerical solutions to $A(r)$. Conventions as in Fig. 1, the profiles being monotonic.

conditions (12) and (40). In particular, we highlight the way $A(r)$ reaches the asymptotic value $A(r \rightarrow \infty) \rightarrow 2 m$.

Figure 3 shows the solutions to the magnetic field $B(r)$, the resulting flux being confined on a ring centered at the origin, its radius increasing as the winding number itself increases. It is also interesting to note that the magnetic

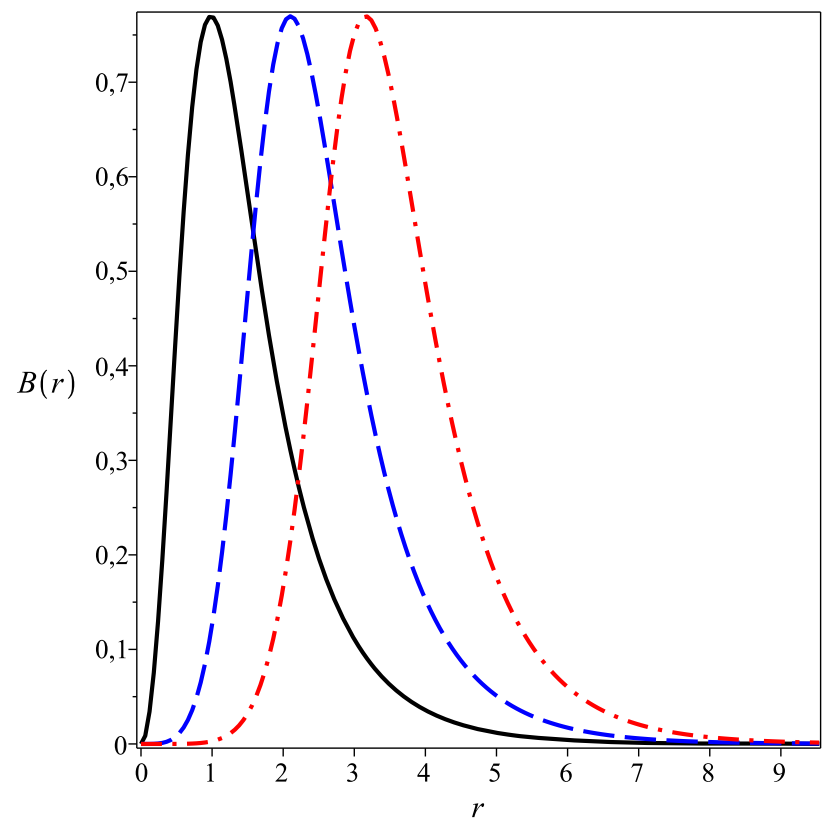

FIG. 3. Numerical solutions to the magnetic field $B(r)$. Conventions as in Fig. 1. The profiles are rings centered at $r=0$.

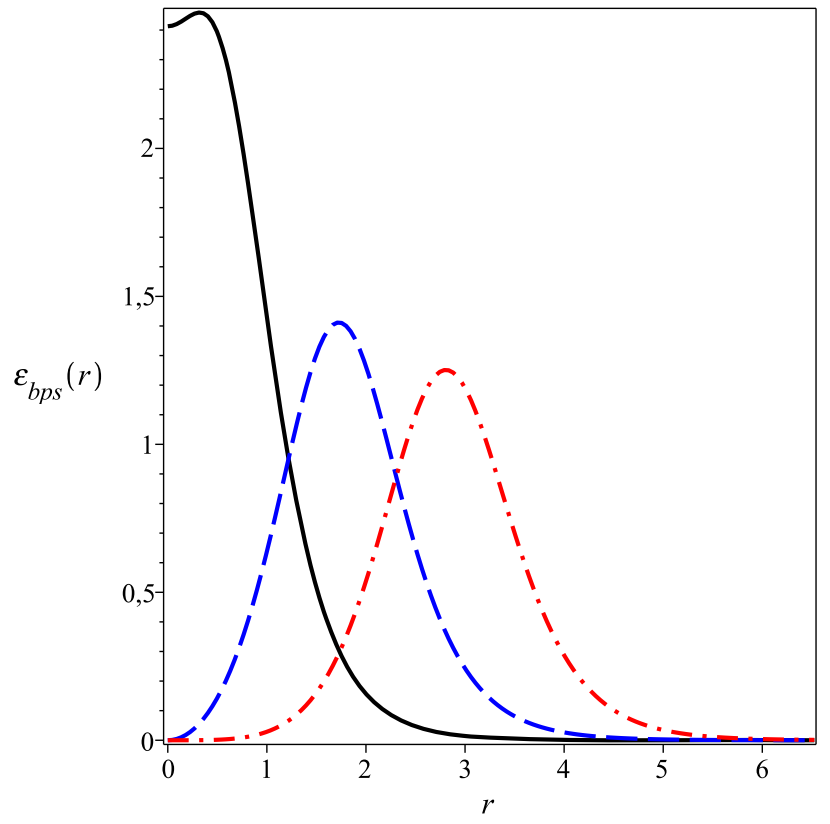

FIG. 4. Numerical solutions to the energy density $\varepsilon_{b p s}(r)$. Conventions as in Fig. 1, $\varepsilon_{b p s}(r=0)$ vanishing for $m \neq 1$.

field vanishes asymptotically, this way fulfilling the finiteenergy requirement $\varepsilon(r \rightarrow \infty) \rightarrow 0$.

In Fig. 4, we depict the profiles to the energy density $\varepsilon_{b p s}(r)$ inherent to the first-order configurations; these solutions also engender rings centered at $r=0$, their radii (amplitudes) increasing (decreasing) as $m$ increases. Here, we point out that $\varepsilon_{b p s}(r=0)$ vanishes for $m \neq 1$. 


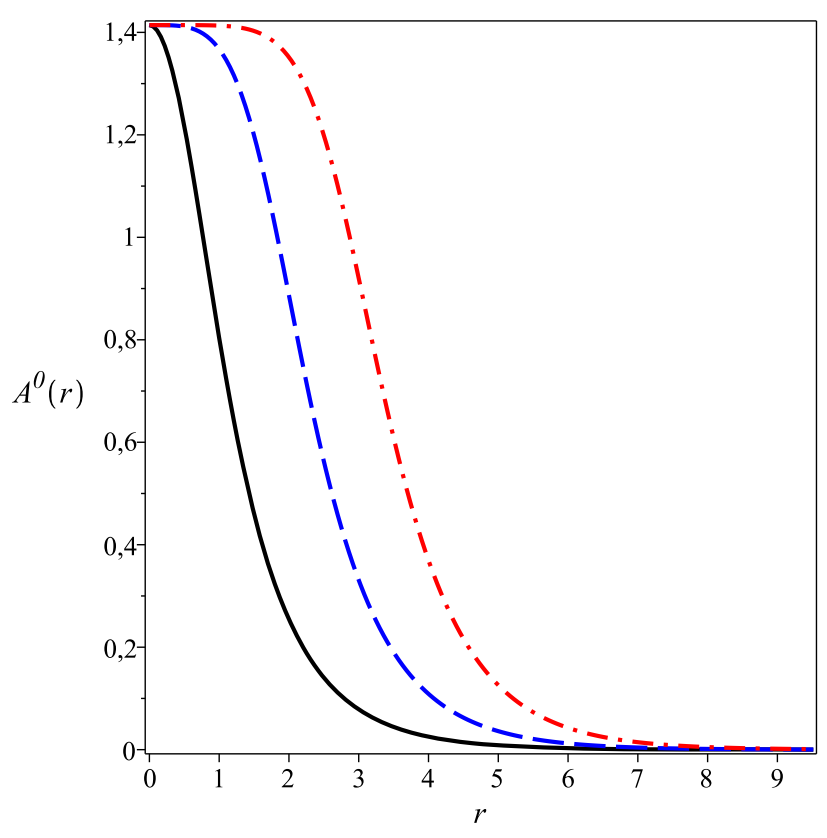

FIG. 5. Numerical solutions to the electric potential $A^{0}(r)$. Conventions as in Fig. 1.

In Figs. 5 and 6, we present the solutions to the electric potential $A^{0}(r)$ and to the electric field $E(r)$ inherent to it, respectively, this last one behaving in the same general way the magnetic field does (i.e., yielding well-defined rings), both $E(r=0)$ and $E(r \rightarrow \infty)$ vanishing identically.

We end this section by studying the Bogomol'nyi limit supporting self-duality. In this sense, we proceed with the linearization of the first-order equations (45) and (46) around the boundary values (12) and (40), for $m>0$

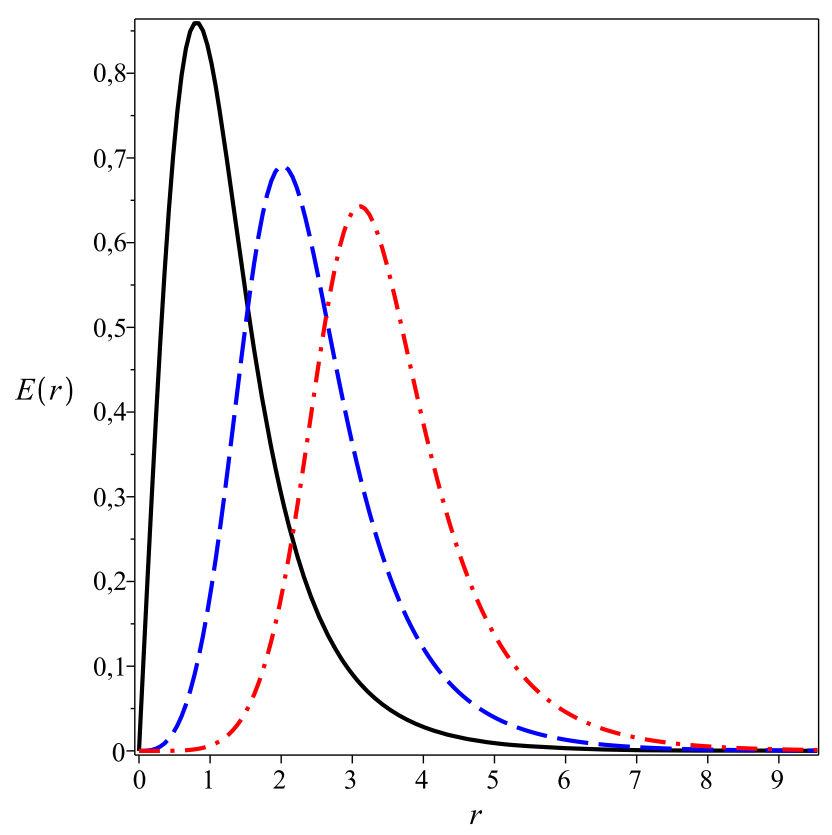

FIG. 6. Numerical solutions to the electric field $E(r)$. Conventions as in Fig. 1, both $E(r=0)$ and $E(r \rightarrow \infty)$ vanishing. (lower signs in the first-order expressions), from which we get the approximate solutions near the origin

$$
\alpha(r) \approx C_{0} r^{m}
$$

and

$$
A(r) \approx \frac{g^{4} h^{2} C_{0}^{2}}{4 k^{2}(m+1)} r^{2(m+1)},
$$

the asymptotic profiles reading

$$
\alpha(r) \approx \frac{\pi}{2}-C_{\infty} e^{-M_{\alpha} r}
$$

and

$$
A(r) \approx 2 m-\frac{g^{2} h}{k} C_{\infty} r e^{-M_{A} r},
$$

$M_{\alpha}=M_{A}=g^{2} h / 2 k$ being the masses of the corresponding bosons (for $h=k=1$ and $g=\sqrt{2}$, both $M_{\alpha}$ and $M_{A}$ equal the unity), the relation $M_{\alpha} / M_{A}=1$ defining the Bogomol'nyi limit. Here, $C_{0}$ and $C_{\infty}$ stand for real positive integration constants to be fixed by requiring the correct behavior at $r=0$ and $r \rightarrow \infty$, respectively. Moreover, given the approximate solutions above, the electric potential $A^{0}(r)$ can be verified to satisfy

$$
A^{0}(r=0)=\frac{g h}{k} \quad \text { and } \quad A^{0}(r \rightarrow \infty) \rightarrow 0,
$$

which do not depend on the winding number $m$; see Fig. 5 .

\section{FINAL COMMENTS AND PERSPECTIVES}

We have investigated the first-order radially symmetric solutions inherent to the $C P(2)$ model in the presence of the Chern-Simons action, from which we have obtained regular solitons saturating a quantized energy lower bound.

We have introduced the overall theory and the conventions inherent to it, focusing our attention on those timeindependent configurations presenting radial symmetry. In the sequel, we have applied the Bogomol'nyi prescription, rewriting the expression for the effective energy in order to introduce a well-defined lower bound (i.e., the Bogomol'nyi bound). The point to be raised is that such a construction was only possible due to a differential constraint involving the potential supporting self-duality.

We have considered separately the cases defined by the two different solutions the additional profile function $\beta(r)$ supports, this way verifying that these two contexts are phenomenologically equivalent, therefore existing as only one effective scenario. We have then solved the corresponding first-order equations numerically by means of the finite-difference algorithm, depicting the resulting profiles we have found this way. We have pointed out the main 
properties the final configurations engender, also studying the Bogomol'nyi limit explicitly.

We highlight that the results we have presented in this work only hold for the radially symmetric structures defined by the map in (10) and (11), being therefore not possible to ensure that the original model supports firstorder solitons outside the radially symmetric proposal, such a question lying beyond the scope of this article.

Ideas regarding future investigations include the search for the nontopological first-order solitons coming from (1) and the development of a well-defined self-dual framework inherent to a $C P(2)$ theory in the presence of both the Maxwell and the Chern-Simons terms simultaneously. These issues are currently under consideration, and we hope provide positive results for an incoming contribution.

\section{ACKNOWLEDGMENTS}

The authors thank CAPES, CNPq, and FAPEMA (Brazilian agencies) for partial financial support.
[1] N. Manton and P. Sutcliffe, Topological Solitons (Cambridge University Press, Cambridge, England, 2004).

[2] E. Bogomol'nyi, Sov. J. Nucl. Phys. 24, 449 (1976); M. Prasad and C. Sommerfield, Phys. Rev. Lett. 35, 760 (1975).

[3] H. B. Nielsen and P. Olesen, Nucl. Phys. B61, 45 (1973).

[4] R. Jackiw and E. J. Weinberg, Phys. Rev. Lett. 64, 2234 (1990); R. Jackiw, K. Lee, and E. J. Weinberg, Phys. Rev. D 42, 3488 (1990).

[5] D. Bazeia, E. da Hora, C. dos Santos, and R. Menezes, Phys. Rev. D 81, 125014 (2010); Eur. Phys. J. C 71, 1833 (2011); D. Bazeia, R. Casana, M. M. Ferreira, Jr., and E. da Hora, Europhys. Lett. 109, 21001 (2015); R. Casana, E. da Hora, D. Rubiera-Garcia, and C. dos Santos, Eur. Phys. J. C 75, 380 (2015); R. Casana, M. M. Ferreira, Jr., E. da Hora, and C. Miller, Phys. Lett. B 718, 620 (2012); R. Casana, M. M. Ferreira, Jr., E. da Hora, and A. B. F. Neves, Eur. Phys. J. C 74, 3064 (2014); R. Casana and G. Lazar, Phys. Rev. D 90, 065007 (2014); R. Casana, C. F. Farias, and M. M. Ferreira, Jr., Phys. Rev. D 92, 125024 (2015); R. Casana, C. F. Farias, M. M. Ferreira, Jr., and G. Lazar, Phys. Rev. D 94, 065036
(2016); L. Sourrouille, Phys. Rev. D 87, 067701 (2013); R. Casana and L. Sourrouille, Mod. Phys. Lett. A 29, 1450124 (2014).

[6] C. Armendariz-Picon, T. Damour, and V. Mukhanov, Phys. Lett. B 458, 209 (1999); V. Mukhanov and A. Vikman, J. Cosmol. Astropart. Phys. 02 (2005) 004; A. Sen, J. High Energy Phys. 07 (2002) 065; C. Armendariz-Picon and E. A. Lim, J. Cosmol. Astropart. Phys. 08 (2005) 007; J. Garriga and V. Mukhanov, Phys. Lett. B 458, 219 (1999); R. J. Scherrer, Phys. Rev. Lett. 93, 011301 (2004); A. D. Rendall, Classical Quantum Gravity 23, 1557 (2006).

[7] A. D'Adda, M. Luscher, and P. D. Vecchia, Nucl. Phys. B146, 63 (1978); E. Witten, Nucl. Phys. B149, 285 (1979); A. M. Polyakov, Phys. Lett. 59B, 79 (1975); M. Shifman and A. Yung, Rev. Mod. Phys. 79, 1139 (2007).

[8] A. Yu. Loginov, Phys. Rev. D 93, 065009 (2016).

[9] R. Casana, M. L. Dias, and E. da Hora, Phys. Lett. B 768, 254 (2017).

[10] R. Casana, M. L. Dias, and E. da Hora, Phys. Rev. D 96, 076013 (2017). 